$<$ Back to results | 1 of 1

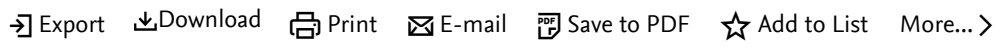

Full Text |View at Publisher|

Document type

Article

Source type

Journal

ISSN

00019240

DOI

10.1017/aer.2020.53

View more $\checkmark$

Aeronautical Journal • Volume 124, Issue 1281, Pages 1740 - 1760 - November 2020

Finite-element modelling of $\mathrm{NiTi}$ shape-memory wires for morphing aerofoils

Wan A. Hamid W.L.H.四, lannucci L.四, Robinson P.四

国 Save all to author list

Department of Aeronautics, Imperial College London, London, United Kingdom

Views count?

View all metrics>

Abstract

Author keywords

Reaxys Chemistry database information

Indexed keywords

SciVal Topics

Metrics

Funding details

Abstract

This paper presents the development and implementation of a user-defined material (UMAT) model for NiTi Shape - Memory Alloy

(SMA) wires for use in LS-DYNA commercial explicit finite - element analysis software. The UMAT focusses on the Shape -

Memory Effect (SME), which could be used for actuation of aerostructural components. The actuation of a fundamental structure

consisting of an SMA wire connected in series with a linear spring was studied first. The SMA thermomechanical behaviour obtained from the finite - element simulation was compared with that obtained from the analytical solution in MATLAB. A further comparison is presented for an SMA-actuated cantilever beam, showing excellent agreement in terms of the SMA stress and strain as well as the tip deflection of the cantilever beam. A mesh sensitivity study on the SMA wire indicated that one beam element was adequate to accurately predict the SMA thermomechanical behaviour. An analysis of several key parameters showed that, to achieve a high recovery strain, the stiffness of the actuated structure should be minimised while the cross-sectional area of the SMA wire should be maximised. The actuation of an SMA wire under a constant stress/load was also analysed. The SMA material model was finally applied to the design of morphing aluminium and composite aerofoils consisting of corrugated sections, resulting in the prediction of reasonably large trailing-edge deflections (7.8-65.9 mm). (c) 2020 Cambridge University Press. All rights reserved.

Author keywords

Keywords: Shape - memory alloy (SMA); LS-DYNA; morphing structures; shape - memory effect (SME); UMAT

Reaxys Chemistry database information (i)

Substances

View all substances (2)

$\mathrm{NiTi}$

Al
Cited by 0 documents

Inform me when this document is cited in Scopus:

Set citation alert >

\section{Related documents}

Characterization of shape memory alloy micro-springs for application in morphing wings

Emiliavaca, A. , De Araujo, C.J. , Souto, C.R.

(2019) Smart Materials and Structures

Self-sensing-based deflection control of carbon fibre-reinforced polymer (CFRP)-based shape memory alloy hybrid composite beams

Lee, S.-H. , Kim, S.-W. (2020) Composite Structures

Optimisation of composite corrugated skins for buckling in morphing aircraft

Shaw, A.D. , Dayyani, I. , Friswell, M.I. (2015) Composite Structures

View all related documents based on references

Find more related documents in Scopus based on:

Authors > Keywords > 


\section{Engineering controlled terms}

Binary alloys; Cantilever beams; MATLAB; Nanocantilevers; Shape-memory alloy; Titanium alloys; Wire

Engineering uncontrolled terms

Actuated structures; Cross sectional area; Explicit finite element analysis; Finite element modelling; Finite element simulations; Fundamental structures; $\mathrm{NiTi}$ shape memory alloys; Thermomechanical behaviour

\section{Engineering main heading}

Finite element method

(i)

Topic name

Morphing; Wing Camber; Ailerons

Prominence percentile

94.689 (i)

Scopus metrics

Views count (2Last updated on 19 May 2021

Views count 2021

Views count 2020

Views count 2012-2021

PlumX metrics ?

Captures

Readers

View PlumX details>

Funding sponsor

See opportunities by MOHE $\lambda$

See opportunities by IIUM $\nearrow$

Funding text

The research is funded by the Ministry of Education Malaysia and IIUM, which is hereby gratefully acknowledged.

References (33)

View in search results format >

$\square$ All Export 召Print 区E-mail 哭 Save to PDF Create bibliography

1 Buehler, W.J., Wiley, R.C.

(1961) The Properties of TiNi and Associated Phases, U.S. Naval Ordnance Laboratory NOLTR 61-75

2 Frotscher, M., Nörtershäuser, P., Somsen, Ch., Neuking, K., Böckmann, R., Eggeler, G. Microstructure and structural fatigue of ultra-fine grained NiTi-stents

(2009) Materials Science and Engineering A, 503 (1-2), pp. 96-98. Cited 24 times. doi: 10.1016/j.msea.2008.02.059

View at Publisher

3 Petrini, L., Migliavacca, $F$.

Biomedical applications of shape memory alloys

(2011) Journal of Metallurgy, 501 (483), pp. 1-15. Cited 268 times.

4 Tobushi, H., Date, K., Miyamoto, K.

Characteristics and development of shape-memory alloy heat engine

(2010) Journal of Solid Mechanics and Materials Engineering, 4 (7), pp. 1094-1102. Cited 23 times. 
$\square$ Banks, R.

The Banks Engine

(1975) Die Naturwissenschaften, 62 (7), pp. 305-308. Cited 4 times.

doi: $10.1007 /$ BF00608890

View at Publisher

6 Kim, H.-I., Han, M.-W., Song, S.-H., Ahn, S.-H.

Soft morphing hand driven by SMA tendon wire

(2016) Composites Part B: Engineering, 105, pp. 138-148. Cited 53 times.

doi: 10.1016/j.compositesb.2016.09.004

View at Publisher

7 Thill, C., Etches, J., Bond, I., Potter, K., Weaver, P.

Morphing skins

(2008) Aeronautical Journal, 112 (1129), pp. 117-139. Cited 434 times.

http://journals.cambridge.org.ezlib.iium.edu.my/AER

doi: $10.1017 /$ S0001924000002062

View at Publisher

8 Barbarino, S., Bilgen, O., Ajaj, R.M., Friswell, M.I., Inman, D.J.

A review of morphing aircraft (Open Access)

(2011) Journal of Intelligent Material Systems and Structures, 22 (9), pp. 823-877. Cited

817 times.

doi: $10.1177 / 1045389 \times 11414084$

View at Publisher

9 Iannucci, L., Evans, M., Irvine, R., Patoor, E., Osmont, D.

(2009) Morphing Wing Design, MCM-ITP Conference

Lille-Grand Palais

10 Jardine, A.Peter, Bartley-Cho, Jonathan, Flanagan, John

Improved design and performance of the SMA torque tube for the DARPA Smart Wing program

(1999) Proceedings of SPIE - The International Society for Optical Engineering, 3674, pp. 260-269. Cited 33 times.

View at Publisher

11 Dong, Y., Boming, Z., Jun, L.

A changeable aerofoil actuated by shape memory alloy springs

(2008) Materials Science and Engineering A, 485 (1-2), pp. 243-250. Cited 56 times. doi: 10.1016/j.msea.2007.08.061

View at Publisher

12 lannucci, L.

Fontanazza Design of morphing wing structures

(2008) 3rd SEAS DTC (System Engineering and Autonomous Systems Defence Technology Centre) Technical Conference

13 Kang, W.-R., Kim, E.-H., Jeong, M.-S., Lee, I., Ahn, S.-M.

Morphing wing mechanism using an SMA wire actuator (Open Access)

(2012) International Journal of Aeronautical and Space Sciences, 13 (1), pp. 58-63. Cited 38

times.

http:/lijass.org/On_line/admin/files/6\%29\%28058-063\%2912-010.pdf

doi: 10.5139/IJASS.2012.13.1.58

View at Publisher 
14 Rim, M., Kim, E.-H., Kang, W.-R., Lee, I.

Development of a shape memory alloy wire actuator to operate a morphing wing

(2014) Journal of Theoretical and Applied Mechanics (Poland), 52 (2), pp. 519-531. Cited 8 times.

http://www.ptmts.org.pl/jtam/index.php/jtam/article/view/v52n2pp519/1844

15 de Almeida, T.C., Santos, O.S., Otubo, J.

Construction of a morphing wing rib actuated by a NiTi wire (Open Access)

(2015) Journal of Aerospace Technology and Management, 7 (4), pp. 454-464. Cited 10

times.

http://www.jatm.com.br/ojs/index.php/jatm/article/download/532/457

doi: $10.5028 / j$ atm.v7i4.532

View at Publisher

16 Iannucci, L.

(2012) Aerofoil Member

US-Patent No. 8,186,631

17 Ruangjirakit, K.

(2013) Polyurethane Corrugated Composites for Morphing Wing Applications

PhD Thesis, Imperial College London, UK

18 Thill, C., Etches, J.A., Bond, I.P., Potter, K.D., Weaver, P.M.

Composite corrugated structures for morphing wing skin applications

(2010) Smart Materials and Structures, 19 (12), art. no. 124009. Cited 128 times. http://iopscience.iop.org/0964-1726/19/12/124009/pdf/0964-1726_19_12_124009.pdf doi: $10.1088 / 0964-1726 / 19 / 12 / 124009$

View at Publisher

19 Terwagne, D., Brojan, M., Reis, P.M.

Smart morphable surfaces for aerodynamic drag control

(2014) Advanced Materials, 26 (38), pp. 6608-6611. Cited 71 times.

http://www3.interscience.wiley.com.ezlib.iium.edu.my/journal/119030556/issue

doi: 10.1002/adma.201401403

View at Publisher

20 Kudva, J.N.

Overview of the DARPA smart wing project

(2004) Journal of Intelligent Material Systems and Structures, 15 (4), pp. 261-267. Cited

271 times.

doi: $10.1177 / 1045389 \times 04042796$

View at Publisher

21 Gao, X., Qiao, R., Brinson, L.C.

Phase diagram kinetics for shape memory alloys: A robust finite element implementation

(2007) Smart Materials and Structures, 16 (6), pp. 2102-2115. Cited 42 times. doi: 10.1088/0964-1726/16/6/013

View at Publisher

22 Birman, V.

Stability of functionally graded shape memory alloy sandwich panels (Open Access)

(1997) Smart Materials and Structures, 6 (3), pp. 278-286. Cited 61 times. doi: $10.1088 / 0964-1726 / 6 / 3 / 006$

View at Publisher 
23 Cross, W.B., Kariotis, A.H., Stimler, F.J.

(1970) Nitinol Characterization Study. Cited 107 times.

NASA CR 1433

24 Alipour, A., Kadkhodaei, M., Ghaei, A.

Finite element simulation of shape memory alloy wires using a user material subroutine: Parametric study on heating rate, conductivity, and heat convection

(2015) Journal of Intelligent Material Systems and Structures, 26 (5), pp. 554-572. Cited 6

times.

http://jim.sagepub.com/

doi: $10.1177 / 1045389 \times 14533431$

View at Publisher

25 Solomou, A.G., MacHairas, T.T., Saravanos, D.A.

A coupled thermomechanical beam finite element for the simulation of shape memory alloy actuators

(2014) Journal of Intelligent Material Systems and Structures, 25 (7), pp. 890-907. Cited 13 times.

doi: $10.1177 / 1045389 \times 14526462$

View at Publisher

26 Tabesh, M., Lester, B., Hartl, D., Lagoudas, D.

Influence of the latent heat of transformation and thermomechanical coupling on the performance of shape memory alloy actuators

(2012) ASME 2012 Conference on Smart Materials, Adaptive Structures and Intelligent Systems, SMASIS 2012, 2, pp. 237-248. Cited 6 times.

ISBN: 978-079184510-3

doi: $10.1115 /$ SMASIS2012-8188

View at Publisher

27 Okabe, Y., Sugiyama, H., Inayoshi, T.

Lightweight actuator structure with SMA honeycomb core and CFRP skins

(2011) Journal of Mechanical Design, Transactions of the ASME, 133 (1), art. no.

011006. Cited 5 times.

doi: $10.1115 / 1.4003139$

View at Publisher

28 Turner, T.L., Cabell, R.H., Cano, R.J., Silcox, R.J.

Development of a preliminary model-scale adaptive jet engine chevron

(2008) AIAA Journal, 46 (10), pp. 2545-2557. Cited 7 times.

http://pdf.aiaa.org/getfile.cfm?

urlX=\%2D\%3CW/\%277D\%2FQKS\%2B\%28S\%20\%23 Ju $020 \% 20 \% 20 \% 0 \mathrm{~A} \&$ urla $=\% 26 \% 2 \mathrm{~A}$

\%22L\%20\%23\%20\%2AC\%0A\&urlb=\%21\%2A\%20\%20\%20\%0A\&urlc=\%21\%2A0\%20\%20

\%0A\&urld=\%21\%2A0\%20\%20\%0A\&urle=\%27\%2B\%22T\%23\%220\%22DU\%20\%20\%20

\%0A

doi: $10.2514 / 1.35939$

View at Publisher

29 Barbarino, S., Pecora, R., Lecce, L., Concilio, A., Ameduri, S., Calvi, E.

A novel SMA-based concept for airfoil structural morphing

(2009) Journal of Materials Engineering and Performance, 18 (5-6), pp. 696-705. Cited 61

times.

doi: $10.1007 /$ s11665-009-9356-3

View at Publisher 
$\square 30$ Sellitto, A., Riccio, A.

Overview and future advanced engineering applications for morphing surfaces by Shape Memory Alloy Materials (Open Access)

(2019) Materials, 12 (5), art. no. 708. Cited 18 times.

https://www.mdpi.com/1996-1944/12/5/708/pdf

doi: $10.3390 / \mathrm{mal} 2050708$

View at Publisher

31 Tanaka, K., Hayashi, T., Itoh, Y., Tobushi, H.

Analysis of thermomechanical behavior of shape memory alloys

(1992) Mechanics of Materials, 13 (3), pp. 207-215. Cited 58 times.

doi: 10.1016/0167-6636(92)90003-V

View at Publisher

32 (2015) Livermore Software Technology Corporation (Lstc), Ls-Dyna. Cited 134 times.

Keyword User's Manual, Volume II Material Models, Version R8.0

33 Epps, J.J., Chopra, I.

In-flight tracking of helicopter rotor blades using shape memory alloy actuators

(2001) Smart Materials and Structures, 10 (1), pp. 104-111. Cited 90 times.

doi: $10.1088 / 0964-1726 / 10 / 1 / 310$

View at Publisher

(C) Copyright 2021 Elsevier B.V., All rights reserved.

\section{About Scopus}

What is Scopus

Content coverage

Scopus blog

Scopus API

Privacy matters

\section{Language}

日本語に切り替える

切换到简体中文

切換到繁體中文

Русский язык
Customer Service

Help

Contact us

ELSEVIER Terms and conditions $\pi$ Privacy policy $\pi$

Copyright (C) Elsevier B.V ז. All rights reserved. Scopus ${ }^{\circledR}$ is a registered trademark of Elsevier B.V.

We use cookies to help provide and enhance our service and tailor content. By continuing, you agree to the use of cookies. 